\title{
Exciton dephasing and biexciton binding in CdSe/ZnSe islands
}

Wagner, Hans Peter; Tranitz, H.-P.; Preis, H; Langbein, Wolfgang Werner; Leosson, Kristjan; Hvam, Jørn Märcher

Published in:

Physical Review B

Link to article, DOI:

10.1103/PhysRevB.60.10640

Publication date:

1999

Document Version

Publisher's PDF, also known as Version of record

Link back to DTU Orbit

Citation (APA):

Wagner, H. P., Tranitz, H-P., Preis, H., Langbein, W. W., Leosson, K., \& Hvam, J. M. (1999). Exciton dephasing and biexciton binding in CdSe/ZnSe islands. Physical Review B, 60(15), 10640-10643.

https://doi.org/10.1103/PhysRevB.60.10640

\section{General rights}

Copyright and moral rights for the publications made accessible in the public portal are retained by the authors and/or other copyright owners and it is a condition of accessing publications that users recognise and abide by the legal requirements associated with these rights.

- Users may download and print one copy of any publication from the public portal for the purpose of private study or research.

- You may not further distribute the material or use it for any profit-making activity or commercial gain

- You may freely distribute the URL identifying the publication in the public portal

If you believe that this document breaches copyright please contact us providing details, and we will remove access to the work immediately and investigate your claim 


\title{
Exciton dephasing and biexciton binding in CdSe/ZnSe islands
}

\author{
H. P. Wagner* \\ Institut für Physik, TU Chemnitz, D-09107 Chemnitz, Germany \\ H.-P. Tranitz and H. Preis \\ Institut Physik II, Universität Regensburg, D-93040 Regensburg, Germany \\ W. Langbein \\ Experimentelle Physik EIIb, Universität Dortmund, D-44221 Dortmund, Germany \\ K. Leosson and J. M. Hvam \\ Research Center COM, Technical University of Denmark, DK-2800 Lyngby, Denmark
}

(Received 17 May 1999)

\begin{abstract}
The dephasing of excitons and the formation of biexcitons in self-organized CdSe/ZnSe islands grown by molecular-beam epitaxy is investigated using spectrally resolved four-wave mixing. A distribution of excitonexciton scattering efficiencies and dephasing times in the range of $0.5-10 \mathrm{ps}$ are observed. This indicates the presence of differently localized exciton states at comparable transition energies. Polarization-dependent measurements identify the formation of biexcitons with a biexciton binding energy of more than four times the bulk value. With decreasing exciton energy, the binding energy slightly increases from 21.5 to $23 \mathrm{meV}$, while its broadening decreases from 5.5 to $3 \mathrm{meV}$. This is attributed to a strong three-dimensional confinement with improving shape uniformity for decreasing exciton energy. [S0163-1829(99)04739-6]
\end{abstract}

Self-organized semiconductor islands providing threedimensional quantum confinement presently attract attention because of their expected superiority for applications in opto-electronic and all-optical devices, where improvements such as a reduction of the laser threshold and higher optical nonlinearities due to the modified density of states are predicted and partly already achieved. ${ }^{1-3}$ The optical properties of such islands have been mainly studied by incoherent methods. ${ }^{4-7}$ Driven by the search for blue-green-emitting semiconductor materials, the fabrication ${ }^{8-13}$ and optical characterization $^{14-18}$ of self-assembled II-VI islands have recently been the subject of intensive research. Only little is known about the coherent properties of these structures, giving information about exciton scattering and biexciton formation. These effects are particularly important in II-VI materials because of their large biexciton binding energy, which strongly influences their stimulated emission. Four-wave mixing (FWM) provides a powerful tool to study the fundamental coherent processes in low-dimensional II-VI semiconductor systems, as already shown in quasitwo-dimensional $^{19,20}$ and quasi-one-dimensional structures. ${ }^{21}$

In this paper we study $\mathrm{CdSe} / \mathrm{ZnSe}$ island structures by femtosecond transient FWM. The excitation-densitydependent dephasing of island excitons and the biexciton binding energy are investigated. The studied $\mathrm{CdSe} / \mathrm{ZnSe}$ island structures were grown by molecular-beam epitaxy (MBE) on a GaAs(001) substrate. Details about the growth and the characterization are given in Ref. 10. The capped $\mathrm{ZnSe}$ island structures were characterized by plan-view transmission electron microscopy (TEM) and high resolution TEM, showing an island density of $5 \times 10^{10} \mathrm{~cm}^{-2}$ and a lateral island size of about $30 \times 15 \mathrm{~nm}^{2}$ elongated along [1 10$]$. Quantitative analysis of the $\mathrm{Cd}$ distribution by a digital analysis of lattice images ${ }^{22}$ shows $\mathrm{Zn}_{1-x} \mathrm{Cd}_{x} \mathrm{Se}$ islands of about $9 \mathrm{ML}$ height with an inhomogeneous $\mathrm{Cd}$ content close to $x=1$ in the island center.

In the FWM experiments, the sample was excited by the signal wave of an optical parametric amplifier pumped by a regenerative Ti-sapphire amplifier, providing 90-fs pulses of $50 \mathrm{~nJ}$ pulse energy at a repetition rate of $300 \mathrm{kHz}$. The pulses are chirp compensated and spectrally shaped to a duration between 120 fs and 3 ps in a pulse shaper. The FWM experiments were performed in reflection geometry with two incident pulses of the directions $\mathbf{k}_{1,2}$, which were colinear $(\uparrow \uparrow)$ or cross-linear $(\uparrow \rightarrow)$ polarized. The focus diameter of the pulses at the $1 / e^{2}$ intensity on the sample was $200 \mu \mathrm{m}$. The FWM signal in the reflected $2 \mathbf{k}_{2}-\mathbf{k}_{1}$ direction was detected time-integrated and spectrally resolved by a combination of a spectrometer and an optical multichannel analyzer as a function of the time delay $\tau$ between $\mathbf{k}_{1}$ and $\mathbf{k}_{2}$. Typical FWM efficiencies were $10^{-5}$. The sample was kept in a helium bath cryostat at a temperature of $5 \mathrm{~K}$.

Microphotoluminescence (PL) measurements (Fig. 1) on the investigated sample reveal a structured band, indicating the presence of individual transitions from localized states with energies distributed around $2.3 \mathrm{eV}$. The strong blueshift compared to the band gap of $1.752 \mathrm{eV}$ in bulk zincblende CdSe (Ref. 23) is due to interdiffusion, strain, and confinement effects as discussed in Refs. 14-18. The FWM experiments are performed using different center wavelengths $\lambda$ of the exciting pulses, indicated by arrows in Fig. 1.

The FWM intensity for $(\uparrow \uparrow)$ configuration (Fig. 2) shows a maximum at $\tau>0$ and a fast quenching for $\tau<0$, typical for the photon-echo response of an inhomogeneously broadened system. For $\tau>0$ a nonexponential decay, slowing 


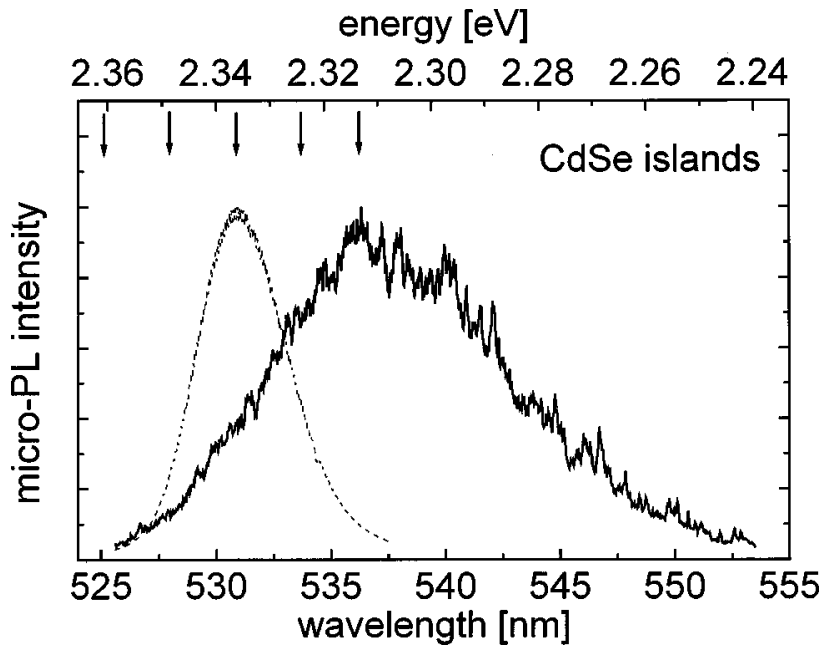

FIG. 1. Micro-PL spectra with $0.5-\mu \mathrm{m}$ resolution of the CdSe quantum island sample at $10 \mathrm{~K}$ excited with $\lambda=514 \mathrm{~nm}$. The dashed line is one spectrum of the FWM excitation pulses. The different center positions are indicated by arrows.

down with increasing decay time, is observed. This reveals a distribution of dephasing times within the excited exciton ensemble, which we attribute to different exciton localization. The FWM signal intensity decreases strongly with decreasing excitation photon energy, a consequence of the decreasing density of states. The smallest excitation photon energy feasible in the FWM experiment corresponds to the maximum of the PL (see Fig. 1). Using a double-exponential fit to the data (thick full line in Fig. 2) we extract two components of different dephasing times $T_{2}$. The homogeneous linewidth $2 \hbar / T_{2}$ of the fast component versus excitation intensity is given in the inset of Fig. 2 for different transition energies. The intensity dependence as well as the zerodensity extrapolated value decreases with decreasing transition energy. Additionally, the weight of the fast component relative to the slow one is decreasing. From these observa-

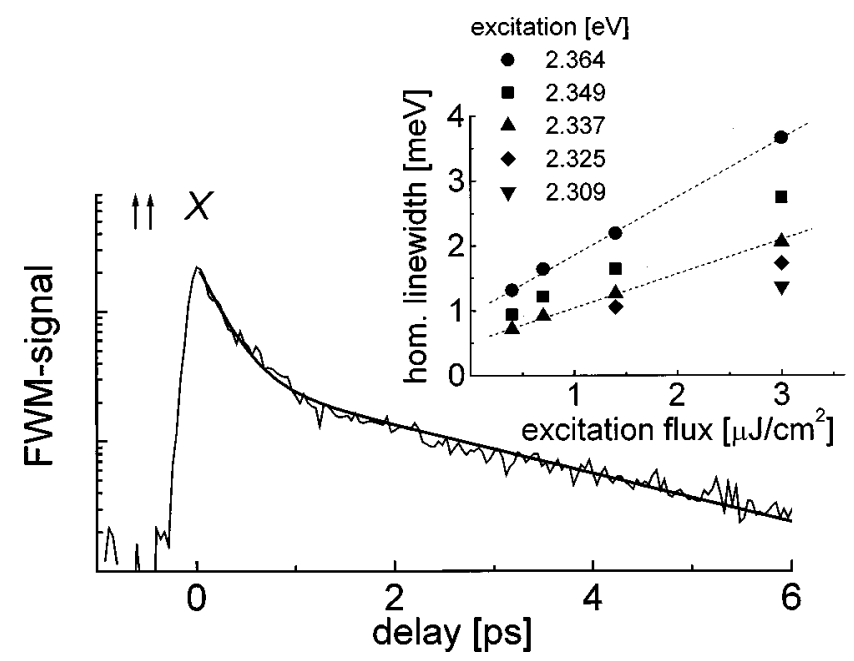

FIG. 2. FWM intensity as a function of the delay time $\tau$ in the $(\uparrow \uparrow)$ configuration, for an excitation energy of $2.325 \mathrm{eV}$ and a total $\mathbf{k}_{1}$ and $\mathbf{k}_{2}$ pulse intensity of $1.4 \mu \mathrm{J} / \mathrm{cm}^{2}$. The inset shows the homogeneous linewidth (full width at half maximum) of the fast component as a function of the pulse intensity for different excitation energies, as labeled.

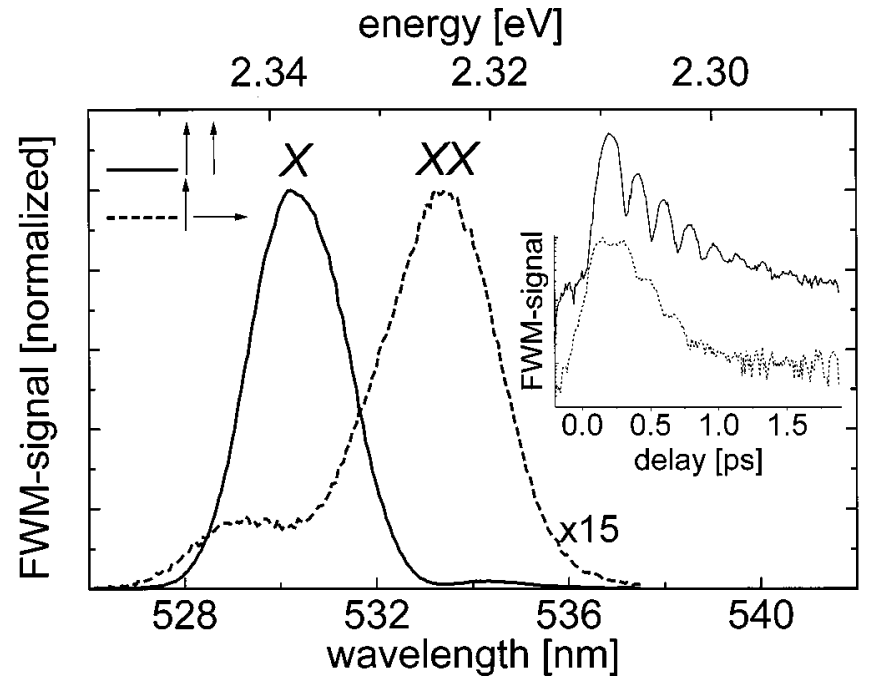

FIG. 3. FWM spectra in the $(\uparrow \uparrow)$ and $(\uparrow \rightarrow)$ configurations at a delay time of $0.4 \mathrm{ps}$, an excitation energy of $2.337 \mathrm{eV}$, and a total pulse intensity of $3 \mu \mathrm{J} / \mathrm{cm}^{2}$. The $(\uparrow \rightarrow)$ data are magnified by a factor of 15 . The inset shows the FWM spectral intensity versus delay time at the position of the biexciton-induced transitions $X X$ for the $(\uparrow \uparrow)$ and $(\uparrow \rightarrow)$ configurations.

tions, we attribute the fast component to more delocalized exciton states, which show more efficient exciton-exciton scattering and in addition phonon-assisted relaxation into lower-lying states. With decreasing transition energy, the fraction of more delocalized exciton states decreases. This is in agreement with the position of the PL maximum, that indicates the transition energy below which radiative recombination is faster than phonon-assisted relaxation. The slow decaying component is accordingly attributed to these more strongly localized excitons. Their homogeneous linewidth decreases slowly with decreasing transition energy ranging from 0.22 to $0.13 \mathrm{meV}$ and shows only a weak density dependence.

The formation of biexcitons is identified by polarizationdependent FWM measurements, for which the selection rules can be derived from four-level ${ }^{24}$ or five-level ${ }^{25}$ Bloch equations. Figure 3 shows FWM spectra for $(\uparrow \uparrow)$ and $(\uparrow \rightarrow)$ polarization configurations. In the $(\uparrow \uparrow)$ configuration the FWM signal is dominated by the ground-state to exciton $(0-X)$ transition (labeled $X)$. For the $(\uparrow \rightarrow)$ configuration the signal mainly originates from the exciton to biexciton $(X-X X)$ transition (labeled $X X$ ), which is spectrally separated from the $(0-X)$ transition by the biexciton binding energy, and is collinear polarized to $\mathbf{k}_{1}$. The strongly suppressed signal at the $(0-X)$ transition shows that exciton to unbound biexciton transitions are weak in these island structures, which is related to the quantization of the biexciton continuum. ${ }^{26}$ The FWM traces at the spectral position of the $X X$ transitions for $(\uparrow \uparrow)$ and $(\uparrow \rightarrow)$ configuration are given in the inset of Fig. 3 . Pronounced oscillations with a period of $T=185 \mathrm{fs}$ arising from the interference between exciton- and biexcitoninduced polarizations are observed, showing the expected phase shift of $\pi$ between $(\uparrow \uparrow)$ and $(\uparrow \rightarrow)$ configurations. ${ }^{27}$ The beats are rather weak in the $(\uparrow \rightarrow)$ configuration due to the small contribution of the unbound biexcitons. The beating period corresponds to a biexciton binding energy of $E_{X X}=22.4 \pm 0.3 \mathrm{meV}$, which is by far larger than the bulk 


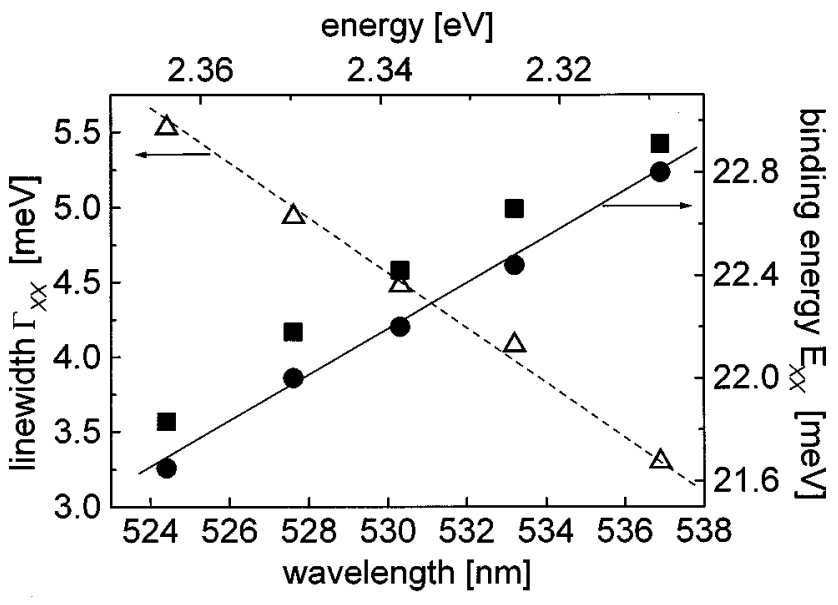

FIG. 4. Biexciton binding energy $E_{X X}$ (full symbols) and inhomogeneous broadening $\Gamma_{X X}$ (open triangles) of the biexcitoninduced $X X$ signal as a function of the exciton transition energy. The circles give the biexciton binding energy $E_{X X}$ determined from the FWM spectra performed with spectrally narrow $\mathbf{k}_{1}$ pulses; the squares show the values obtained from the FWM beat period.

CdSe biexciton binding energy of about $4 \mathrm{meV},{ }^{18}$ indicating a strong three-dimensional quantum confinement in the islands. This is in agreement with experiments on similar structures showing biexciton binding energies of about 20 $\mathrm{meV}$ (Refs. 17 and 18) and also to theoretical and experimental results for spherical CdSe quantum dots in glasses. ${ }^{28,29} \mathrm{We}$ observe a decrease of the oscillation period and thus an increase of the biexciton binding energy of about $5 \%$ with decreasing exciton transition energy, plotted as filled squares in Fig. 4. The observed increase of the biexciton binding energy is attributed to the stronger spatial localization in the energetically deeper localized states, similar to what has been found in other localized systems. ${ }^{30}$

A direct determination of the biexciton binding-energy distribution from the FWM spectra can be achieved by selecting a narrow distribution of $(0-X)$ transitions with a spectrally narrow $\mathbf{k}_{1}$ pulse. ${ }^{31} \mathrm{FWM}$ spectra for the $(\uparrow \rightarrow)$ configuration and a $\mathbf{k}_{1}$ spectral width of $1 \mathrm{meV}$ are given in Fig. 5 , where the scattered light intensity from the $\mathbf{k}_{1}$ pulse indicates the energy of the selected $(0-X)$ transition. The FWM spectra were normalized by the spectral intensity of the spectrally broad $\mathbf{k}_{2}$ pulse in order to isolate the spectral shape of the biexcitonic FWM response. The extracted biexciton binding energies (filled circles in Fig. 4) show a slight increase with decreasing exciton transition energy, similar to the data extracted from the beat period. Furthermore, the $(X-X X)$ transition exhibits an additional broadening, which is decreasing from 5.5 to $3.3 \mathrm{meV}$ with decreasing $(0-X)$ transition energy (Fig. 5). Such an additional broadening of the biexciton binding energy has been observed in localized quasi-two-dimensional systems ${ }^{26,31}$ and is explained as an inhomogeneous broadening due to a nonuniform shape of the localization potentials for excitons even at comparable transition energies. The observed decrease of the broadening (Fig. 4) is accordingly interpreted as improved shape unifor-

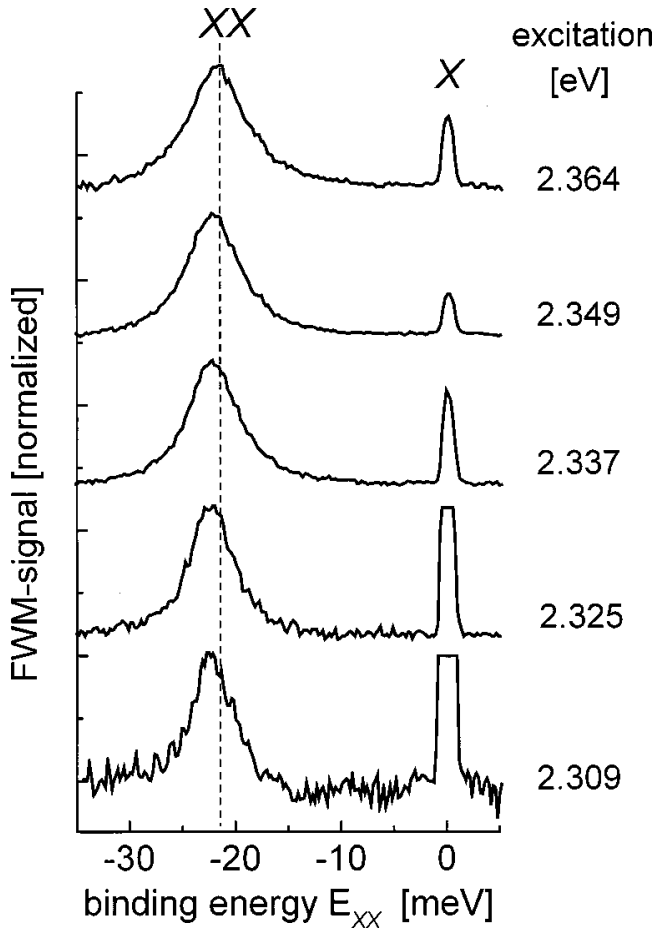

FIG. 5. FWM spectra at $\tau=0.4$ ps using a spectrally narrow $\mathbf{k}_{1}$ pulse $(\Delta E=1 \mathrm{meV})$ obtained at various excitation wavelengths for the $(\uparrow \rightarrow)$ configuration. The energetic distance between the $\mathbf{k}_{1}$ pulse and the biexciton-induced $X X$-signal directly gives the biexciton binding energy.

mity of strongly localized states, which are less influenced by the surrounding potential landscape due to their smaller extension. This is consistent with the observed increase of the biexciton binding energy, also indicating stronger localization.

In summary, the low-temperature dephasing of excitons in the investigated self-organized $\mathrm{ZnSe}$ capped $\mathrm{CdSe}$ island structures shows a distribution of dephasing times due to differently localized excitons. The faster decaying components, which show a large exciton-exciton scattering efficiency, are attributed to weakly localized excitons that can relax by phonon emission into more strongly localized states. The slower components, on the other hand, are attributed to these more strongly localized states, and show a weaker exciton-exciton scattering efficiency. The localized biexcitons in the structures have binding energies of $22 \mathrm{meV}$, more than four times the bulk value, indicating a strong threedimensional confinement. Since biexcitons with such high binding energies are stable at room temperature they are important for applications in all-optical or opto-electronic devices. With decreasing transition energy, the biexciton binding energy is slightly increasing and its inhomogeneous broadening is decreasing, which is attributed to shrinking size and increasing shape uniformity of the localized states.

This work was supported by the Deutsche Forschungsgemeinschaft and by the Danish Ministries of Research and Education in the framework of the Center for Nanostructures. 
* Author to whom correspondence should be addressed. Present address: Universität Regensburg, Physik II, Universitätsstraße 31, D-93040 Regensburg, Germany. Fax: 49 9419434226. Electronic address: Hans-Peter.Wagner@physik.uni-regensburg.de (or) HP.Wagner@physik.tu-chemnitz.de

${ }^{1}$ Y. Arakawa and A. Yariv, IEEE J. Quantum Electron. 22, 1887 (1986).

${ }^{2}$ Y. Z. Hu, M. Lindberg, and S. W. Koch, Phys. Rev. B 42, 1713 (1990).

${ }^{3}$ D. Bimberg et al., Phys. Status Solidi 194, 159 (1996).

${ }^{4}$ O. Brandt, K. Ploog, R. Bierwolf, and M. Hohenstein, Phys. Rev. Lett. 68, 1339 (1992).

${ }^{5}$ C. W. Snyder, B. G. Orr, D. Kessler, and L. M. Sander, Phys. Rev. Lett. 66, 3032 (1991).

${ }^{6}$ M. Grundmann et al., Phys. Rev. Lett. 74, 4043 (1995).

${ }^{7}$ J. A. Prietro, G. Armelles, T. Vtzmeier, F. Briones, J. C. Ferrer, F. Peiro, A. Cornet, and J. R. Morante, Phys. Rev. Lett. 80, 1094 (1998)

${ }^{8}$ Hyun-Chui Ko, Doo-Cheol Park, Y. Kawakami, S. Fujita, and S. Fujita, Appl. Phys. Lett. 70, 3278 (1997).

${ }^{9}$ Z. Zhu, E. Kurtz, K. Arai, Y. F. Chen, D. M. Bagnall, P. Tomasini, F. Lu, T. Sekiguchi, T. Yao, T. Yasuda, and Y. Segawa, Phys. Status Solidi B 202, 827 (1997).

${ }^{10}$ H. Preis, S. Kaiser, S. Blümel, S. Miethaner, S. Bauer, K. Fuchs, and W. Gebhardt, in Proceedings of the Microscopy of Semiconducting Materials Conference 1999, edited by A. G. Cullis (IOP, Oxford, in press).

${ }^{11}$ H. Kirmse, R. Schneider, M. Rabe, W. Neumann, and F. Henneberger, Appl. Phys. Lett. 72, 1329 (1998).

${ }^{12}$ N. N. Ledentsov, I. L. Krestnikov, M. V. Maximov, S. V. Ivanov, S. L. Sorokin, P. S. Kopev, Zh. I. Alferov, D. Bimberg, and C. M. Sotomayor Torres, Appl. Phys. Lett. 69, 1343 (1996).

${ }^{13}$ D. Hommel, K. Leonardi, H. Heinke, H. Selke, K. Ohkawa, F. Gindele, and U. Woggon, Phys. Status Solidi B 202, 835 (1997).

${ }^{14}$ S. H. Xin, P. D. Wang, Aie Yin, M. Dobrowolska, J. L. Merz, and J. K. Furdyna, Appl. Phys. Lett. 69, 3884 (1996).

${ }^{15}$ F. Flack and N. Samarth, V. Nikitin, P. A. Crowell, J. Shi, J.
Levi, and D. D. Awschalom, Phys. Rev. B 54, R17 312 (1996).

${ }^{16}$ K. Leonardi, H. Heinke, K. Ohkawa, and D. Hommel, Appl. Phys. Lett. 71, 1510 (1997).

${ }^{17}$ F. Gindele, C. Märkle, U. Woggon, W. Langbein, J. M. Hvam, K. Leonardi, K. Ohkawa, and D. Hommel, J. Cryst. Growth 184/ 185, 306 (1998).

${ }^{18}$ F. Gindele, U. Woggon, W. Langbein, J. M. Hvam, K. Leonardi, D. Hommel, and H. Selke, Phys. Rev. B 60, 8773 (1999).

${ }^{19}$ H. P. Wagner, R. Maier, A. Schätz, W. Langbein, and J. M. Hvam, Phys. Rev. B 57, 1791 (1998).

${ }^{20}$ H. P. Wagner, W. Langbein, and J. M. Hvam, Phys. Rev. B 59, 4584 (1999).

${ }^{21}$ H. P. Wagner, W. Langbein, J. M. Hvam, G. Bacher, T. Kümmell, and A. Forchel, Phys. Rev. B 57, 1797 (1998).

${ }^{22}$ A. Rosenauer, S. Kaiser, T. Reisinger, J. Zweck, and W. Gebhardt, Optik (Stuttgart) 102, 63 (1996).

${ }^{23}$ S. Fujita, Y. Wu, Y. Kawakami, and S. Fujita, J. Appl. Phys. 72, 5233 (1992).

${ }^{24}$ K. Bott, O. Heller, D. Bennhardt, S. Cundiff, P. Thomas, E. Mayer, G. Smith, R. Eccleston, J. Kuhl, and K. Ploog, Phys. Rev. B 48, 17418 (1993).

${ }^{25}$ E. J. Mayer, G. O. Smith, V. Heuckeroth, J. Kuhl, K. Bott, A. Schulze, T. Meier, S. W. Koch, and P. Thomas, R. Hey, and K. Ploog, Phys. Rev. B 51, 10909 (1995).

${ }^{26}$ W. Langbein, P. Borri, and J. M. Hvam, TOPS, edited by D. S. Citrin (Optical Society of America, Washington, D.C., 1998), Vol. 18, p. 11.

${ }^{27}$ T. F. Albrecht, K. Bott, T. Meier, M. Koch, S. T. Cundiff, J. Feldmann, W. Stolz, P. Thomas, S. W. Koch, and E. O. Göbel, Phys. Rev. B 54, 4436 (1996).

${ }^{28}$ K. I. Kang, A. D. Kepner, S. V. Gaponenko, S. W. Koch, Y. Z. $\mathrm{Hu}$, and N. Peyghambarian, Phys. Rev. B 48, 15449 (1993).

${ }^{29}$ U. Woggon, O. Wind, W. Langbein, O. Gogolin, and C. Klingshirn, J. Lumin. 59, 135 (1994).

${ }^{30}$ W. Langbein and J. M. Hvam, Phys. Rev. B 59, 15405 (1999).

${ }^{31}$ W. Langbein, J. M. Hvam, M. Umlauff, H. Kalt, B. Jobst, and D. Hommel, Phys. Rev. B 55, R7383 (1997). 